A N N A L E S Annales de Bretagne et des Pays de l'Ouest

\title{
AUBERT Gauthier, Le président de Robien, gentilhomme et savant dans la Bretagne des Lumières
}

\section{Frédérique Pitou}

\section{(2) OpenEdition}

Édition électronique

URL : https://journals.openedition.org/abpo/1574

DOI : $10.4000 /$ abpo. 1574

ISBN : 978-2-7535-1487-4

ISSN : 2108-6443

Éditeur

Presses universitaires de Rennes

Édition imprimée

Date de publication : 20 septembre 2002

Pagination : 162-165

ISBN : 978-2-86847-768-2

ISSN : 0399-0826

\section{Référence électronique}

Frédérique Pitou, «AUBERT Gauthier, Le président de Robien, gentilhomme et savant dans la Bretagne des Lumières ", Annales de Bretagne et des Pays de l'Ouest [En ligne], 109-3 | 2002, mis en ligne le 20 septembre 2004, consulté le 22 juillet 2022. URL : http://journals.openedition.org/abpo/1574 ; DOI : https://doi.org/10.4000/abpo.1574 
apparaît donc comme une étude sur le phénomène de l'immigration, centrée à juste titre sur les réseaux - regroupement familial compris -. La particularité d'une partie de ses sources est indiscutable, mais nous disposons à l'inverse, dans le cadre " métropolitain ", de possibilités à ce jour quasi inexploitées dans la perspective de l'étude de la mobilité : je pense en particulier aux archives notariales et aux archives judiciaires. Il est donc raisonnable d'espérer que la méthode inspirera des chercheur(e)s de l'ancienne France et les incitera à se lancer dans l'exploration de nos migrations intérieures encore si peu connues.

Alain CROIX

Gauthier AUBERT, Le président de Robien, gentilhomme et savant dans la Bretagne des Lumières, Rennes, PUR, coll. "Art et Société ", 2001, 396 p.

Un examen attentif du portrait de Christophe-Paul de Robien sur la couverture du livre peut-il indiquer que, malgré l'énergie consacrée par ce gentilhomme breton à montrer, puis à faire reconnaître la supériorité de son rang, la vraie vie, pour lui, était ailleurs?

C'est finalement ce que montre l'ouvrage de Gauthier Aubert sur le président de Robien, résultat d'une traque systématique et fructueuse qui l'a amené partout où pouvaient être conservées des traces du célèbre magistrat et de son action. L'objectif est de cerner un personnage dans son milieu avec la volonté farouche d'en établir l'éventuelle originalité ou au contraire d'en constater le conformisme. C'est donc muni des enseignements fournis par de très importantes lectures que G. Aubert a entrepris l'étude de trois ensembles documentaires : les écrits savants du président de Robien, ce qui reste des archives familiales des Robien de l'Ancien Régime et les documents classiques de l'histoire sociale tels que registres fiscaux, lettres, minutes notariales, afin de retrouver des traces du personnage. Mais il ne s'est pas arrêté là, pour cerner le personnage dans son siècle, il a regardé vers d'autres curieux ou amateurs bretons du $\mathrm{xVIII}^{\mathrm{e}}$ siècle, ainsi que vers les élites urbaines en général, dont il a dépouillé les inventaires sur 15 ans (1741-1755) dans cinq villes (Rennes, Nantes, Saint-Malo, Brest, Quimper), soit 454 dossiers...

Bien qu'elle soit considérée en 1781 par Chérin comme "noblesse maigre ", la famille de Robien apparaît régulièrement dans les archives dès le xiII ${ }^{\mathrm{e}}$ siècle, comme une moyenne noblesse, essentiellement composée d'hommes d'armes; jusqu'au début du XVI ${ }^{\mathrm{e}}$ siècle, elle reste enracinée dans le pays de Quintin. Elle franchit une nouvelle étape en entrant au service de la reine de France, Claude, fille d'Anne de Bretagne; au milieu du xvI ${ }^{\mathrm{e}}$ siècle, la francisation du nom (et l'abandon de l'ancienne forme Rocbihan), témoigne de l'ascension sociale de la famille. Celle-ci s'explique également par la confirmation de la fidélité à la monarchie française, et en particulier à Henri IV. Christophe I' ${ }^{\text {er }}$ (1573-1625) reçoit le collier de l'ordre de Saint-Michel, est fait premier gentilhomme de la chambre et gouverneur de la ville et du comté de Quintin. Son petit-fils Sébastien entre au Parlement en 1655 comme conseiller originaire. Il effectue là le même choix qu'une partie de la noblesse militaire médiévale qui tente de conserver son influence à l'époque où le caractère administratif de la monarchie s'accentue : le Parlement est le lieu du pouvoir provincial. La famille n'abandonne pas l'épée : le premier parlementaire de la famille n'est qu'un cadet, son frère aîné se destinait à la carrière des armes. La situation est inversée à la génération suivante, 
lorsque le fils aîné, Paul, devient également conseiller originaire, puis achète en 1706 une charge de président à mortier. Le fils de Paul, Christophe-Paul, né en 1698 (demeuré " anonime de Robien " jusqu'à son baptême à 16 ans), devient conseiller en 1720 , président à mortier en 1724, comme son père, comme son beau-père et deux de ses beaux-frères, comme le sera son fils à sa mort en 1756 .

Chistophe-Paul est donc un président à mortier du Parlement de Bretagne, que G. Aubert montre soucieux d'affirmer son rang; le bon usage de l'héraldique et de la généalogie sert à cela. G. Aubert passe en revue différentes occurrences du blason des Robien, depuis la décoration d'un plat à barbe en laque de Chine, jusqu'à celle des façades du château du Plessis de Kaër; il figure même sur le retable de l'église paroissiale de Pluvigner où les armes de Robien ont temporairement remplacé celles du roi... Le président aimait mettre autour de ses armes un mortier associé au manteau d'hermine et à la couronne du duc de Bretagne, manifestation de l'attachement à la grandeur du Parlement de Rennes, héritier de la justice ducale. De la même façon, plus qu'au titre de marquis qui lui est quelquefois donné au XVIII ${ }^{\mathrm{e}}$ siècle, Christophe-Paul de Robien tient bien davantage à celui de baron de Kaër; c'est l'indice d'une prise de distance à l'égard des modèles nobiliaires versaillais, ses références sont la Bretagne médiévale dans laquelle les barons sont l'équivalent des pairs.

Le rang de Christophe-Paul est naturellement lié à sa fortune. Cette " grande fortune bretonne " est connue par un acte de partage réalisé après sa mort en 1764; elle s'élève à plus d'un million de livres. Le premier poste (près de $80 \%$ de la fortune totale) est constitué par les biens ruraux et en premier lieu par les treize seigneuries et leurs dépendances ainsi que par plusieurs domaines congéables. Viennent ensuite la charge de président à mortier (plus de 100000 livres), puis les biens mobiliers (estimés à près de 78000 livres, ce qui est particulièrement élevé en raison du " cabinet " inventorié dans ce poste), les immeubles urbains, les contrats et billets. Le président est ainsi propriétaire d'un ensemble de terres groupées en trois endroits de la province : la région de Quintin, le Trégor et la région d'Auray où ont été faites les acquisitions du XVIII siècle (notamment celle de la seigneurie du Kaër, en 1727). Robien gère ses biens comme les autres grands propriétaires ruraux, profitant sans excès de la rente foncière, mais il n'envisage pas de tirer parti de la terre en dehors du cadre seigneurial, d'où ces procès auquel il consacre, selon G. Aubert, beaucoup de temps et d'énergie, sa volonté de jouer les premiers rôles suscitant une véritable révolte de ses vassaux.

Au Parlement de Bretagne, Christophe-Paul de Robien fit une longue carrière (1720-1756). Il y entre jeune (à 22 ans), il accède rapidement au mortier et se maintient en fonction jusqu'à sa mort. G. Aubert voit en lui un conseiller puis un président à l'activité moyenne et doute presque de son intérêt pour les questions judiciaires : d'une part, parmi les nombreux manuscrits qu'il a laissés, presque aucun ne comporte de réflexion sur le métier de juge ou la pratique judiciaire, à l'exception de la proposition faite en 1738 (c'est l'époque de d'Aguesseau) pour réformer la coutume et la jurisprudence bretonnes, d'autre part, sa bibliothèque (si tant est que l'on puisse distinguer l'apport réel de Christophe-Paul de celui de son père ou de son fils..., et G. Aubert se montre, comme à son habitude, d'une très grande prudence à cet égard) est celle d'un magistrat ordinaire, montrant peu d'intérêt pour le droit pénal. On peut imaginer, avec G. Aubert, que si le président ne se retire pas du Palais après un certain nombre d'années dans le mortier, contrairement à ce que font nombre de ses confrères, c'est bien à cause du prestige de l'institution (et peut-être parce qu'il espérait continuer à servir ses intérêts personnels). 
Ce n'est sans doute pas pour y jouer un rôle politique; G. Aubert le montre fidèle à la monarchie lorsqu'il siège au Parlement, mais presque méprisant dans son refus de siéger aux États de Bretagne où il pourrait cependant représenter le roi (d'une manière générale, les parlementaires ne siègent pas aux États, mais les trois plus anciens présidents à mortier sont commis par le roi pour le représenter) : sa fierté d'être au plus haut niveau de la hiérarchie administrative de la province ne lui fait pas accepter l'idée de n'être qu'un banal agent du pouvoir royal. À propos des idées politiques du président, G. Aubert se pose toutes les questions possibles, évoque toutes les pistes, et ce n'est sans doute pas un hasard si le paragraphe qu'il leur consacre voit fleurir les "sans doute " et les " peut-être ". La grande question est bien sûr celle des libertés bretonnes; Christophe-Paul se réfère à la Bretagne médiévale et aux belles heures du duché, et s'il critique la conspiration de Pontcallec, c'est qu'il fait du Parlement le protecteur des privilèges provinciaux. Mais la vision qu'il a du Parlement ne va pas vraiment plus loin, car l'heure n'est pas venue : Christophe-Paul occupe une position intermédiaire entre ses ancêtres, fidèles sujets de la monarchie et son fils nettement critique et favorable à La Chalotais.

Le président de Robien affirme également son rang dans ses résidences. Il partage l'essentiel de son temps entre son hôtel rennais et ses châteaux campagnards. Si l'hôtel rennais est " sans splendeur ", ses propriétaires disposent à proximité d'un beau jardin à la française avec un bâtiment à l'italienne dont l'aménagement fastueux lui vaut le surnom de Trianon et dont la vocation est expressément de recevoir la compagnie durant l'été. À la campagne, on serait tenté d'imaginer le président dans le magnifique château de Robien; or G. Aubert montre que sa construction est vraisemblablement plus tardive et serait l'œuvre de son fils. Le président à la campagne est plutôt mis en scène dans le cadre du Plessis de Kaër, jardin, bois, et même bateaux de plaisance sont autant d'éléments de sa vie de château.

Après avoir étudié autant de témoignages variés de la place éminente du président de Robien dans la société de son temps, G. Aubert s'interroge sur son rayonnement réel. Il trouve à ce personnage " orgueilleux et même un peu autoritaire " peu de vrais amis (dont le poète Desforges-Maillard) et beaucoup d'ennemis. Ses interventions dans la société bretonne, en dehors de son rôle au parlement, semblent limitées : peu d'actions charitables à son actif dans les paroisses, pas de rôle véritable dans la création d'institutions d'éducation auxquelles il se serait cependant intéressé; Robien au sommet de la société bretonne, supporte mal la comparaison avec les plus grands seigneurs du royaume. Il est vrai que le président a choisi, avec sa collection, d'autres façons de rayonner dans la société de son temps.

Le président de Robien collectionne tout ce qui se collectionnait en Europe depuis la Renaissance. G. Aubert commence l'étude de son cabinet par l'histoire naturelle (la collection serait composée dans ce domaine de près de 8000 objets, minéraux végétaux, animaux). Examinant les pièces conservées, leur classement, les légendes et les commentaires de Robien, il tente de faire la part de ce qui le rattache aux curieux de la Renaissance et de ce qui en fait un homme des Lumières, connaisseur de la science moderne. Il s'attache ensuite aux " ouvrages de l'art ", parmi lesquels les monnaies et médailles (plus de 2500 pièces c'est sans doute la collection la plus importante de Bretagne), les pierres gravées (plus de 200), les antiques, les objets d'outre-mer puis à la collection artistique, étudiée par type d'œuvres, dessins, gravures, peintures, avec ce paradoxe qu'alors que les dessins montrent un goût pour le grand genre et l'Italie, la collection de peinture est plutôt orientée vers les écoles du Nord. Pour chacune 
de ces dernières collections, G. Aubert examine autant ce qu'il peut percevoir des intentions du Président (volonté de posséder quelques exemplaires de chaque école) et de ses goûts (goût pour le réalisme, voire pour le pittoresque) ainsi que des occasions d'acquisition des différentes pièces.

Ses collections, associées à la bibliothèque, font du cabinet de Robien (dont G. Aubert nous restitue la topographie) un espace tourné vers la science et le travail, susceptible d'accueillir des savants du cru ou de recevoir la visite ponctuelle de voyageurs plus lointains qui l'intègrent à la république de la curiosité; le cabinet de Robien fait en effet partie des plus fameux cabinets cités en 1742 par Dezallier d'Argenville. Robien est dans ce domaine-là plus chanceux que dans celui de projets académiques, ses tentatives pour créer une telle institution à Rennes, en 1727 puis 1738 se soldant par un échec dont G. Aubert présente de multiples causes sans réelle conviction, préférant incriminer "le déficit de culture urbaine " des élites rennaises plutôt que le facile " désert culturel breton ", renvoyant en dernière analyse à la rédaction finalement solitaire de la Description historique, topographique et naturelle de la Bretagne... au départ liée aux projets académiques du président et qui a pour objectif de faire entrer la Bretagne dans le champ du savoir. C'est sans doute la réputation de cet ouvrage qui explique son élection à l'Académie de Berlin en tant que membre externe en 1755.

Il faut remercier l'éditeur d'avoir accepté les 243 illustrations qui enrichissent le livre de G. Aubert parmi lesquelles, entre bien d'autres, on a plaisir à citer de véritables trouvailles pour le propos de l'ouvrage, comme Le déjeuner au Plessis-de-Käer attribué à Pierre Angillis, qui dit tout sur les activités des Robien près de la rivière d'Auray, comme l'allégorie familiale signée Huguet et réalisée pour l'Essay d'histoire généalogique, plus mystérieuse au premier regard, mais dont G. Aubert fait une lecture très fine de même que du Trompe-l'oeil à la médaille d'Anne de Bretagne par Jean-Valette-Penot exécuté en 1748. La distance prise par Robien avec la monarchie absolue se lirait dans la présence d'un petit personnage représentant un marchand de poisson, contribution du président aux poissonnades visant la maîtresse de Louis XV... Une superbe Tête de jeune homme de Boticelli montre que la collection comprend des œuvres d'artistes italiens de grand renom mais le seul dessin retrouvé signé Robien représente un paysage montrant l'influence des écoles du Nord.

La lecture de ce grand livre érudit témoigne de l'ampleur du travail de l'historien, il restitue également la complexité de la pensée des hommes des Lumières, surtout lorsque celles-ci sont pré-encyclopédiques, nobiliaires et de surcroît, bretonnes.

Frédérique PITOU

Bernard GAINOT, 1799, un nouveau jacobinisme? La démocratie représentative, une alternative à Brumaire, Paris, CTHS, 2001, 542 p., $31 €$.

Jean-Clément Martin rappelle dans la préface de ce volumineux ouvrage que le bicentenaire n'a pas tout dit et que des pans entiers de la Révolution restent à réévaluer. Bernard Gainot aborde ici la question quelque peu délaissée du néojacobinisme. Ce néologisme désigne le projet démocrate d'aménagement progressif des institutions républicaines da ns le sens d'une plus large participation des citoyens à la vie politique, projet qui s'épanouit en l'an VII lors de la dernière belle saison du Directoire. L'étude se fonde sur les journaux, les dis- 